\title{
Effects of Self-Shadowing on Nonconservative Force Modeling for Mars-Orbiting Spacecraft
}

\author{
Erwan Mazarico* and Maria T. Zuber \\ Massachusetts Institute of Technology, Cambridge, Massachusetts 02139 \\ and \\ Frank G. Lemoine $₫$ and David E. Smith $₫$ \\ NASA Goddard Space Flight Center, Greenbelt, Maryland 20771
}

\begin{abstract}
DOI: $\underline{10.2514 / 1.41679}$
Modeling improvements of nonconservative forces affecting Mars-orbiting spacecraft are presented in this study. Recent high-resolution gravity fields enable the recovery of smaller signals in the radio tracking data, previously obscured by mismodeled gravitational anomalies. In particular, the estimation of the atmospheric drag experienced by the spacecraft benefits from the new force models. More precise calculations of the spacecraft cross-sectional areas entering the equations for the atmospheric drag and direct solar radiation pressure are possible by accounting for the interplate self-shadowing of the spacecraft physical model. The relevant surface areas can change by as much as $20 \%$ on average, and the effects can be very variable within one orbit $( \pm 10 \%)$. The benefits of these updated models are assessed with two spacecraft, the Mars Odyssey and the Mars Reconnaissance Orbiter. The changes in the nonconservative forces can significantly impact the reconstructed spacecraft trajectory and the estimated model parameters depend on the spacecraft geometry and orbit. The atmospheric density estimated by the Mars Odyssey is much improved with self-shadowing applied to the solar radiation, but improvements to both the drag force and the solar radiation are important in this case of the Mars Reconnaissance Orbiter.
\end{abstract}

\section{Introduction}

$\mathbf{I}^{\mathrm{N}}$ N RECENT years, several NASA Mars orbiter missions provided valuable tracking data with the aim to improve the knowledge of the Martian gravity field. The Mars Global Surveyor (MGS) was tracked extensively over nearly 10 years. The Mars Odyssey (launched in early 2001) and Mars Reconnaissance Orbiter (MRO, launched in late 2005) are still operational and continue to be tracked nearly continuously.

The designs of the three spacecraft included movable high-gain antennae, so that acquisition of radio tracking and downlink of telemetry to Earth would not interfere with science data collection. In contrast, the sparse tracking of the ESA Mars Express spacecraft is due to its fixed high-gain antenna on the spacecraft bus. These recent NASA missions considerably improved the spatial resolution of the estimated Martian gravity field compared with earlier models [1-3]. The level of the unmodeled gravity anomalies decreased considerably as a result, and the nongravitational forces have a more noticeable impact on the adjusted trajectory. This enables the estimation of previously unadjustable parameters, such as the atmospheric drag [3-ㅡ]

Because the nonconservative forces are small and generally of the same order of magnitude, improvements of the orbit reconstruction and of the confidence in the adjusted force parameters depend on better modeling of all of them. The results of modeling efforts focused on the spacecraft cross section calculations are presented. Better models of atmospheric density structure or seasonal surface temperatures are of course important for the estimation of the nonconservative forces, but the surface area affects all these small forces.

Presented as Paper 7201 at the AIAA/AAS Astrodynamics Specialist Conference and Exhibit, Honolulu, HI, 18-21 August 2008; received 28 October 2008; revision received 18 February 2009; accepted for publication 6 March 2009. Copyright (C) 2009 by Erwan Mazarico. Published by the American Institute of Aeronautics and Astronautics, Inc., with permission. Copies of this paper may be made for personal or internal use, on condition that the copier pay the $\$ 10.00$ per-copy fee to the Copyright Clearance Center, Inc., 222 Rosewood Drive, Danvers, MA 01923; include the code 0022-4650/ $09 \$ 10.00$ in correspondence with the CCC.

*Currently NASA Postdoctoral Program Fellow, NASA Goddard Space

Flight Center; Erwan.M.Mazarico@nasa.gov.

${ }^{\dagger}$ Solar System Exploration Division.
The current modeling scheme of the nonconservative forces is first presented and its limitations are addressed. The new algorithm used to accurately estimate the spacecraft cross section is explained in detail, followed by an assessment of its computational performance and its implementation in the precision orbit determination (POD) workflow.

Sample trajectory arcs of the Mars Odyssey and MRO spacecraft are used to show the differences that arise with the new modeling, including changes in the cross-sectional areas and nonconservative accelerations. The effects on a larger scale, covering most of the missions of both spacecraft, are presented as well. The more detailed study of the MRO shows how the modeling improvements can affect, for the better, the ability to recover small signals in the tracking data. The physical configuration of the MGS spacecraft and the phasing of the orbit were such (early afternoon) that the ensuing self-shadowing is much smaller than for the other two spacecraft, and so it is not discussed in further detail.

Finally, the modeling of the planetary radiation pressure acceleration modeling is discussed, both in terms of practical difficulties and of potential future beneficial improvements.

In all this work, the POD program "GEODYN II" developed at NASA Goddard Space Flight Center (GSFC) [7] was used. The orbit of the spacecraft is forward integrated using accurate force models, and the radio tracking data are compared with values predicted from the computed trajectory. The differences (residuals) are used along with the partial derivatives of all the adjustable parameters in order to obtain the parameters' values for a new iteration until convergence is attained. Here, contrary to other studies where the focus was gravity field estimation, only the initial state, measurement biases, and of course the atmospheric drag and solar radiation scale factors, are adjusted. The density model for the Martian upper atmosphere is a modified Stewart-Culp model [4], which was used in previous Mars orbiters' radio tracking data studies at NASA GSFC $[\underline{2}, \underline{5}, \underline{6}]$.

\section{Model Description}

\section{A. Principle and Algorithm}

Although spacecraft with simple geometries can be very useful for geodetic studies (Starlette, LAGEOS), many spacecraft missions with more general scientific instruments have more complex 
physical configurations. In addition to one or two large movable solar panels, recent NASA Mars orbiters had a movable high-gain antenna, which allowed the return of unprecedented amounts of data because the spacecraft could be tracked over long periods of time without interfering with the operations of the science instruments. As a result of the three-axis attitude stabilization and the temporal positions of the target body, the sun and the Earth, the spacecraft goes through a wide variety of geometries. From the point of view of POD, these attitude considerations can potentially shift the center of gravity from its nominal position and modify the magnitude and direction of the nonconservative forces by way of changing the relevant cross-sectional area of the spacecraft. Here the focus is the latter.

Atmospheric drag and solar radiation pressure have a significant effect on satellite orbits, and they must be included in orbit models [8,9]. The modeling of the albedo (reflected short-wave solar flux) and thermal (planetary long-wave flux) radiation pressures came later because of the smaller magnitude of those accelerations and to its higher computational cost $[10,11]$. In addition to a priori models for the atmospheric density model and the albedo and thermal flux, the cross-sectional area of the spacecraft seen from the relevant direction enters the equations defining those nonconservative accelerations. The simplest assumption is that the spacecraft behaves as a cannonball (i.e., constant cross section from any direction), and although this could be acceptable for the direct solar radiation with a three-axis stabilized spacecraft pointing its solar arrays towards the sun at all times, it would probably fail for the other accelerations. In the case of the MRO, for instance, the average cross section for the solar radiation is $35 \mathrm{~m}^{2}$, whereas it is only $15 \mathrm{~m}^{2}$ for the atmospheric drag. To answer this need to realistically evaluate the cross section of the spacecraft from any direction, the use of spacecraft macromodels was implemented in GEODYN II [12]. A macromodel is a set of plates defined by their surface area, their normal in the spacecraft frame, and radiation-related parameters (principally diffuse and specular reflectivity, as well as emissivity). At each time step of the trajectory integration, these plates are oriented in space according to external attitude information (supplied in the form of quaternions). The cross section is calculated simply by scaling the plate area by the dot product of the plate normal with the appropriate viewing unit vector, and only the plates having their front facing the direction considered are used. For this reason, the movable parts such as antennae and solar arrays are modeled by two identical but opposite plates, front and back. This was a tremendous improvement for the radiation pressure accelerations because it can distinguish the contributions of the different plates and, thus, calculate the total acceleration based on their individual radiative characteristics. Macromodels (without self-shadowing) have been applied in the analysis of the MGS and the Odyssey [2, $, \underline{5}, 6]$.

\section{Self-Shadowing}

However, defining the plates simply by their normal prevents the macromodel from capturing the actual three-dimensional configuration of the spacecraft. In particular, the ordering of the plates given a viewing direction cannot be computed. With complex spacecraft geometries, the obscuration of some plates by others is common and can significantly change the spacecraft cross-sectional areas. This interplate interaction is referred to as "self-shadowing." The goal of this paper is to present how the macromodel approach was used as a starting point to improve the modeling of the nonconservative forces and how these improvements were used. Simplified implementations of self-shadowing effects have been used by the MRO navigation team in also recent Mars gravity field publications [3] . The main selfimposed constraint was to be compatible with the current implementation of small force modeling in the GEODYN II orbit determination program used at NASA GSFC. The self-shadowing algorithm would return only numerical cross-section values of individual plates defined in the macromodel. This makes it easy to switch on or

\footnotetext{
D. Highsmith, personal communication, 2008.
}

off the use of self-shadowing (for sanity check purposes during development). But more important, it ensures that the parts of the code dealing with summing the total force and calculating the partials with respect to the different reflectivities were not changed [13].

A different definition of the spacecraft geometry was designed, which would use the same kinds of plates as previously but with the addition of nodes defined by their Cartesian coordinates in the spacecraft body frame, and of node links defining polygons (i.e., plates, which need to be convex). A center of rotation for each movable panel also needs to be defined by three Cartesian coordinates. Although the rotated normal vector is independent of the point around which it is rotated, the attached nodes are not. These centers of rotation actually correspond to the gimbals of the solar panels and high-gain antenna.

\section{Algorithm}

The geometry of the spacecraft is described in a simple text file, indicating the location of the nodes constituting the various polygonal plates, the corresponding index of those plates in the GEODYN II framework, the location of the gimbals of the movable plates, and the plate normal vectors for sanity checks. After reading the geometry file, arrays defining the plate polygons in the spacecraft body frame are created. At each time step, all the plates are oriented in space given the available attitude information: movable plates are first rotated around their gimbals, then all the plates are rotated from the spacecraft frame to the inertial frame used by GEODYN II for its trajectory integration. All the plates are then projected onto a plane perpendicular to the input viewing vector. Those two-dimensional projected polygons are assigned two properties: a "depth" (larger values meaning closer to the observer) and a "sign," initialized to +1 . The depth is used for knowing which of two plates is in front and which is behind. The sign is needed for the intersections, to keep track of their intersection order: positive for the polygon being the result of an even number of intersections (including none) and negative for an odd number. This is critical, when you (correctly) subtract the areas of intersections (order 1), you have to add back the areas of the intersections of intersections (order 2), see Fig. 1. Also, these areas are not to be added to the total area because the crosssectional area of each individual plate needs to be output; instead, they are added to the "deepest" order 0 polygon that created it. The intersection polygons are assigned the depth of the "closest" of the two polygons that created it. They are kept in a growing set of polygons, so that they can reintersect with others. The algorithm in pseudocode is shown next.

\section{B. Computational Cost}

In an effort to reduce the computational load of the algorithm, the plates forming the spacecraft are imposed to be convex, which considerably simplifies the intersection computations. This also

\section{Algorithm 1 Pseudocode algorithm for the self-shadowing calculations}

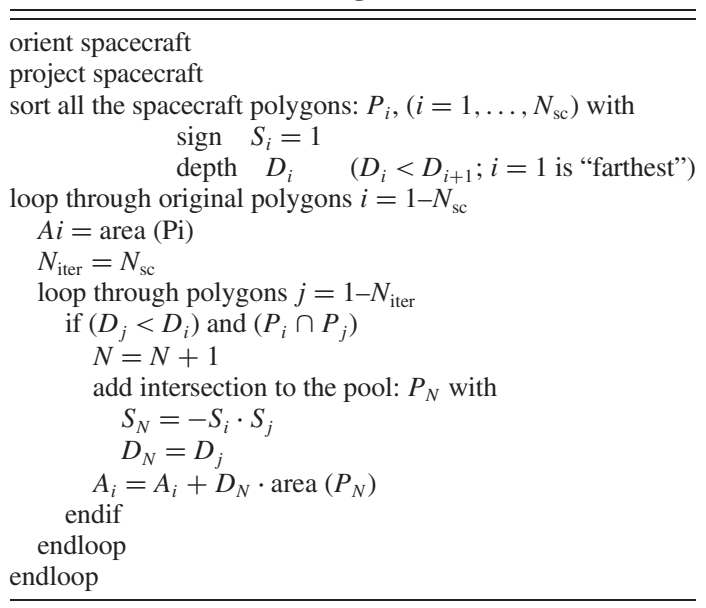




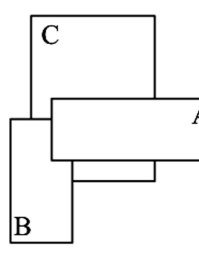

view from the input vector

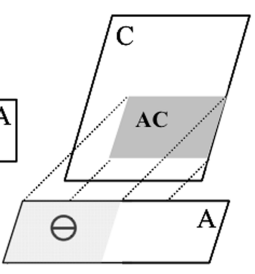

shadowing of $\mathrm{C}$ by $\mathrm{A}$ (to be subtracted)

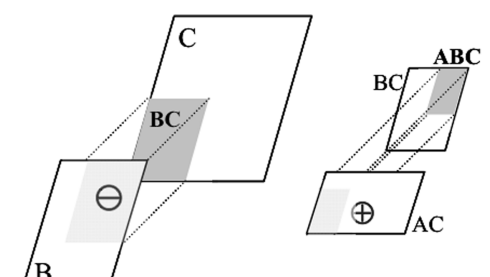

intersection of the two shadowed areas of C (to be added)

Fig. 1 Schematic of a two-level intersection.

seems to be natural, and so far all the macromodels, based on simplified spacecraft geometries, were made of rectangular or octagonal shapes.

Nevertheless, as illustrated in the algorithm description previously, this is significantly more complex than orienting a plate normal and calculating a dot product. The implementation of the algorithm directly inside of GEODYN II considerably slows the orbit determination process, and the algorithm itself is the bottleneck. It is called at each time step multiple times (once for solar radiation, once for atmospheric drag, and potentially numerous times for albedo), a process that is iterated in order to find the best fit to the radio tracking data. In a typical two-day arc with the MRO, it can be called around 100,000 times (without the albedo).

\section{Adopted Operational Method}

Consequently, the self-shadowing calculation part was separated from the orbit determination part. Given that the initial state adjustments are generally very small compared with the distances to the sun and the planet (meters compared with at the very least tens of kilometers) and to the total velocity ( $\mathrm{cm} / \mathrm{s}$ compared with $\mathrm{km} / \mathrm{s}$ ), it is a very good approximation to consider that the self-shadowed plate cross sections will not vary appreciably between the a priori trajectory and the converged trajectory. Thus, the self-shadowing calculations just need to be performed once.

The program MATLAB $®$ was used to rewrite the algorithm and do the calculations for the whole Mars Odyssey and MRO mapping and extended phases (24 April 2002 to 20 September 2007 and 3 November 2006 to 14 March 2008, respectively). The spacecraft position and attitude information were obtained using the SPICE [14] bindings to MATLAB [15]. In the event of missing attitude data, quaternions were interpolated, using pattern copying for large gaps. Statistics on the execution times for the Mars Odyssey (5 polygons, corresponding to the 10 plates of the GEODYN II macromodel) and MRO (6 polygons, corresponding to 12 GEODYN II plates) are given in Table 1, based on more than 100,000 calls to the algorithm.

GEODYN II was then modified to read in a file with time-tagged plate areas corresponding to a given number of directions (currently, only atmospheric drag or solar radiation). When calculating $t$ he nonconservative accelerations, the cross-section values were obtained with a simple linear interpolation. The full implementation into the main GEODYN II code base is currently under testing and should be completed very shortly.

\section{Effect of Self-Shadowing on Spacecraft Accelerations}

First, examples of the effects of self-shadowing on the force modeling for each spacecraft is presented. The following sections will have a broader perspective, discussing time periods of several years. Here, just a few orbits in selected arcs are discussed (Fig. 2). The chosen arcs are: a one-day arc of the Mars Odyssey (starting 19 July $20031119 \mathrm{hrs}$ ), and a two-day arc of the MRO (starting 11 October 20072256 hrs).

The top panel of Fig. 2 presents the direct effect of self-shadowing in the cross section of the spacecraft to be used for the computation of the nonconservative forces. Self-shadowing clearly affects both spacecraft very differently and each force as well. For the Mars Odyssey, the self-shadowing in the drag direction is low (approximately 5\%) and nearly constant over an orbit, whereas the impact on solar radiation is larger (maximum above 10\%) and more variable. This results in solar radiation changes (compared with the nonshadowing case) that are comparable in magnitude to the total atmospheric drag force (bottom panels of Fig. 2). Small mismodelings in the solar radiation (average magnitude approximately $7.10^{-8} \mathrm{~m} \cdot \mathrm{s}^{-2}$ ) can thus greatly impact the recovery of the drag acceleration (average magnitude approximately $5.10^{-9} \mathrm{~m} \cdot \mathrm{s}^{-2}$ ).

The complex geometry of the MRO accounts for the very different character of the cross-section changes. The larger size of the highgain antenna and the presence of two solar panels (just one on the Mars Odyssey) result in significantly larger effects on the cross sections (top right). The modeling of self-shadowing for the atmospheric drag force is more important, as both acceleration changes are large relative to the total magnitude (lower right, average magnitudes are respectively approximately $4.10^{-8} \mathrm{~m} \cdot \mathrm{s}^{-2}$ and approximately $10^{-8} \mathrm{~m} \cdot \mathrm{s}^{-2}$ for solar radiation and drag).

In the next two sections, the impact of the changes in acceleration magnitudes and orbital signatures (owing to large self-shadowing effect asymmetry within an orbit) on the reconstructed orbits and the values of important arc parameters that can be relevant to scientific investigations are investigated.

\section{Mars Odyssey}

The Mars Odyssey spacecraft began its primary mission in February 2002 and is still in operation. It is in an orbit very similar to the ones of the MGS and the MRO: sun-synchronous (approximately $1700 \mathrm{hrs}$ ), nearly-circular, polar and a periapsis frozen near the south pole. However, its periapsis altitude (approximately $390 \mathrm{~km}$ ) is slightly more than MGS (approximately $370 \mathrm{~km}$ ) and significantly higher than MRO (approximately $250 \mathrm{~km}$ ). This has made atmospheric density measurements with the Mars Odyssey more challenging. Unlike MGS and MRO, it only has one solar array and a small high-gain antenna. Figure $\underline{3}$ shows the spacecraft seen from the three directions related to the nonconservative forces: from the sun, from the drag direction, and from nadir (in August 2004, DOY $_{2002} \sim 965$, i.e., day of year 2002 around 965). Because the solar panel is nearly edge-on along the spacecraft velocity, the resulting self-shadowing is small. The situation is quite different

Table 1 Execution times of the self-shadowing algorithm

\begin{tabular}{lccccc}
\hline \hline & \multicolumn{4}{c}{ Mean execution time of one algorithm call, ms } \\
\hline Spacecraft & Number of arcs & Minimum & Mean & Maximum & Standard \\
Odyssey & 1908 & 6.2 & 39.3 & 159 & 22.1 \\
MRO & 409 & 22.9 & 69.3 & 95.6 & 6.8 \\
\hline \hline
\end{tabular}



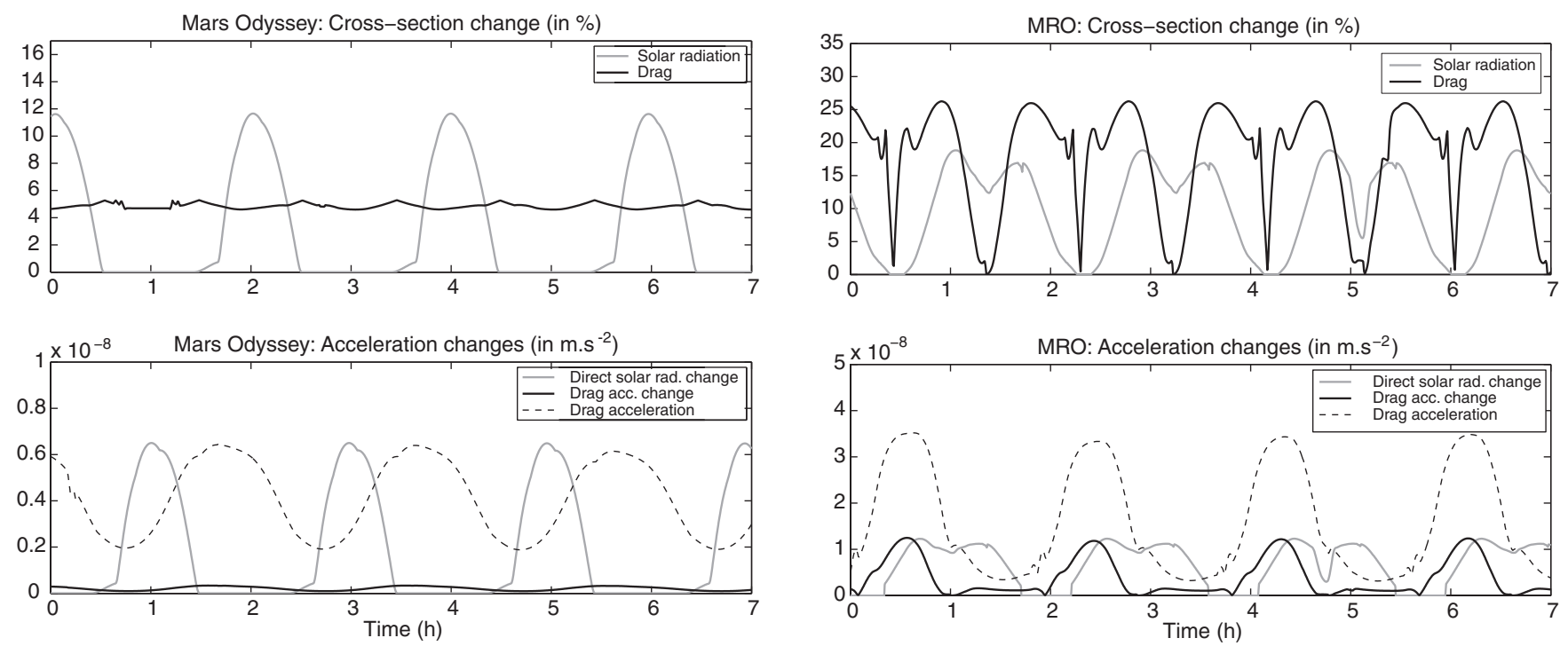

Fig. 2 Relative cross-section changes and acceleration differences due to self-shadowing.

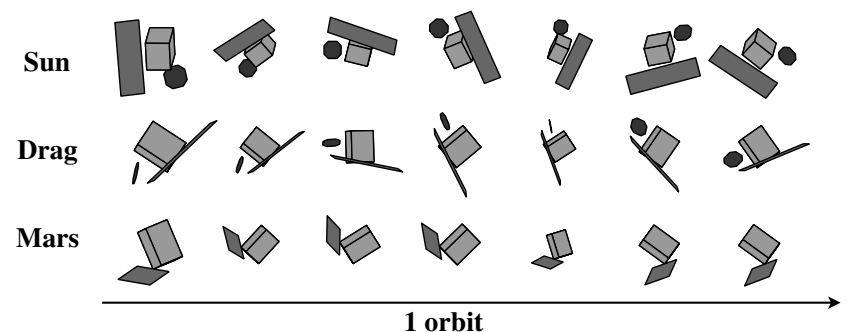

Fig. 3 Mars Odyssey spacecraft geometry over one orbit.

when viewed from the sun: the solar panel shadows a large part of the bus but only during about half an orbit. This results in a larger selfshadowing impact on the total cross section, in terms of average value but also of a much larger range. This has two important consequences: the use of self-shadowing for the drag will not have a strong effect on the reconstructed orbits because its effect can be entirely taken care of by a simple scaling (increase) of the drag coefficient parameter; the "signature" of the solar radiation on the other hand changes significantly, and can have notable consequences in the force parameter adjustments performed by GEODYN II. Figure 4 illustrates this. The effect of the self-shadowing on the spacecraft cross section is shown in percent for solar radiation (gray) and atmospheric drag (black), with the mean as a thick line and the minimum and maximum as dotted lines. The observed secular variations in the solar radiation cross-section changes are closely related to the orbit geometry, and especially the viewing angle of the orbit from the sun $\left(\beta_{\odot}\right.$, measured in degrees, angle between orbit normal and Mars-sun line). When that angle is small, the orbit is perpendicular to the sun, and the solar panel is constantly facing the sun, off the spacecraft bus.

Figure 5 shows the orbital changes, in terms of root-mean-square (rms) total position differences, induced by the use of self-shadowing for either the solar radiation or the atmospheric drag. At the Mars Odyssey's orbiting altitude, the solar radiation is more than one order of magnitude larger than the atmospheric drag, and the resulting orbital changes are of the comparable relative magnitude. A better modeling of the solar radiation is the most critical. For the same reasons, it is particularly challenging to obtain reliable estimates of that scale factor to the a priori atmospheric density. Slight mismodelings of the other forces can account for many times the magnitude of the atmospheric drag acceleration. One drag coefficient per trajectory arc was adjusted, each about one day long (that duration is driven primarily by the interval between angular momentum desaturation maneuvers). The results are noisier than
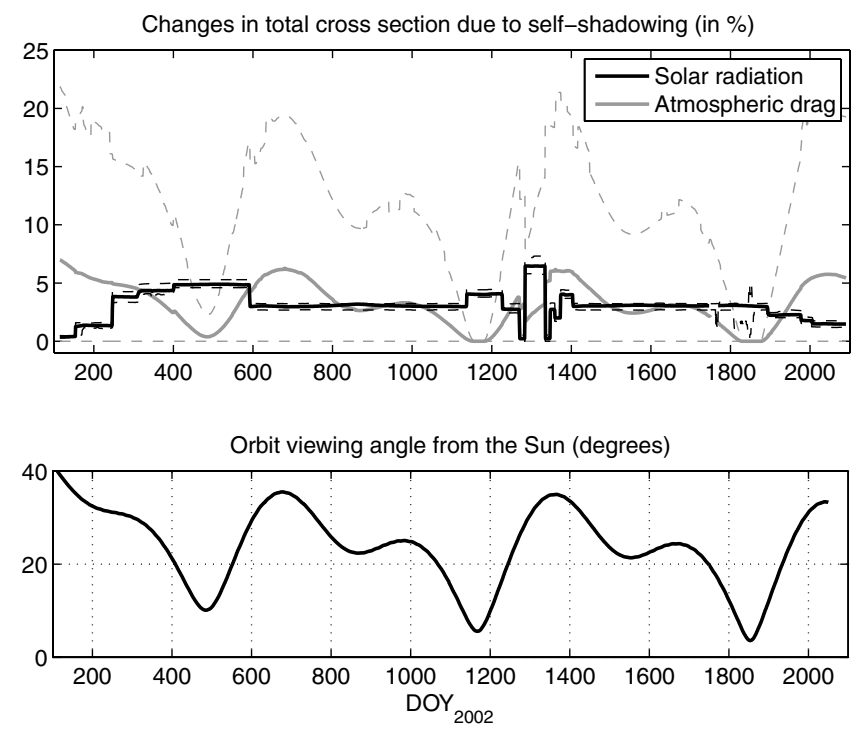

Fig. 4 Self-shadowing effect on cross section for the Mars Odyssey, and orbit viewing angle from the sun $\left(\beta_{\odot}\right)$, over approximately 5.5 years.

with the four- to five-day arcs used in previous studies [6], but the benefits of self-shadowing modeling are clearer. The use of selfshadowing for the solar radiation significantly impacts the obtained values, as shown in Fig. 6. Four modeling strategies are compared: no self-shadowing (black filled circles), self-shadowing on the atmospheric drag only (light gray dots), on the solar radiation only (gray filled circles), and on both (dark gray dots). In particular, two

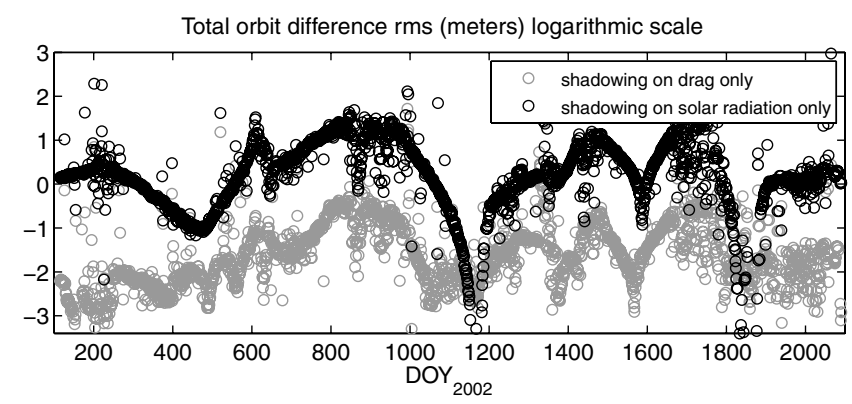

Fig. 5 Mars Odyssey orbit differences due to the modeling of selfshadowing for solar radiation and atmospheric drag. 


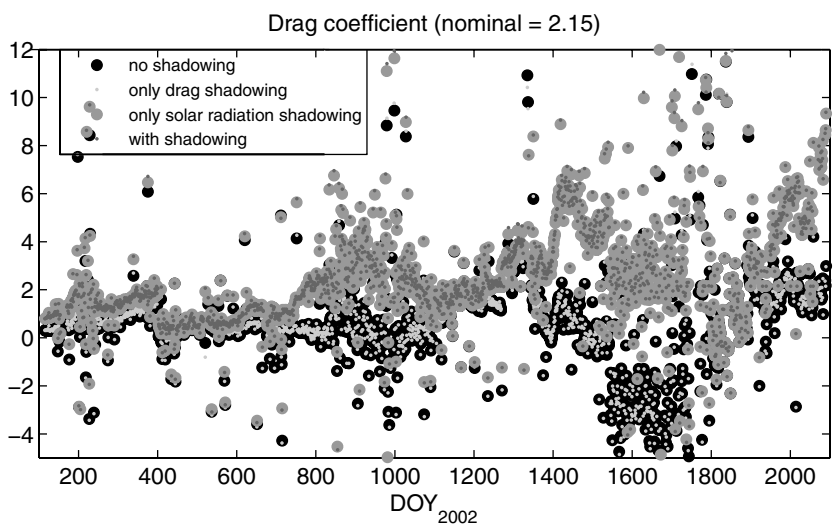

Fig. 6 Time series of the obtained drag coefficients by the Mars Odyssey with different modeling approaches.

periods had very poor drag recovery before (many negative values): DOY $_{2002} \sim 900-1100$ and DOY $_{2002} \sim 1600-1900$. The viewing geometry from Earth was not favorable to radio tracking observations (orbit nearly face-on, resulting in a small line-of-sight component of the velocity), and the atmospheric density was generally low (northern spring-northern summer seasons, decreasing or low solar activity). The use of the self-shadowing modeling was a dramatic improvement there, with retrieved values now physically acceptable. This shows how the improved modeling of one nonconservative force can considerably help improve the retrieval of scientifically valuable parameters in other nonconservative forces.

\section{Mars Reconnaissance Orbiter}

After a short aerobraking phase, the MRO, launched in August 2005, started its primary mission in September 2006 in a $250 \times 320 \mathrm{~km}$ polar orbit. At those altitudes, the atmospheric drag force is much larger than on the similar orbits of MGS and the Mars Odyssey and enables the estimation of the atmospheric density much more frequently than what was possible before [16], that is, one drag coefficient estimated every two orbits (approximately $4 \mathrm{~h}$ ). The sun synchronicity of the orbit, with a local mean solar time of approximately $230 \mathrm{hrs}$ and $1430 \mathrm{hrs}$, leads to atmospheric sampling of those solar longitude only. The larger relative difference between altitudes at apoapsis and periapsis also means that the integrated global density measurements are more weighted towards the southern hemisphere than for MGS and the Mars Odyssey. In addition to carrying two large solar panels, MRO carries a large highgain antenna, because of the large amounts of data that need to be transmitted. This leads to significant self-shadowing in the sun and velocity directions, as can be seen in Fig. 7 for one orbit in mid-November 2006 (DOY $_{2002} \sim 320$ ). The evolution of the selfshadowing over the first year of mission is shown in Fig. $\underline{\text { 8. Similarly }}$ to Fig. 4, the effects on the solar radiation pressure and the atmospheric drag are shown in blue and red respectively; results for the nadir direction, for the albedo radiation pressure, are also shown in lighter tone. The period $\mathrm{DOY}_{2006}=430-550$ stands out. An

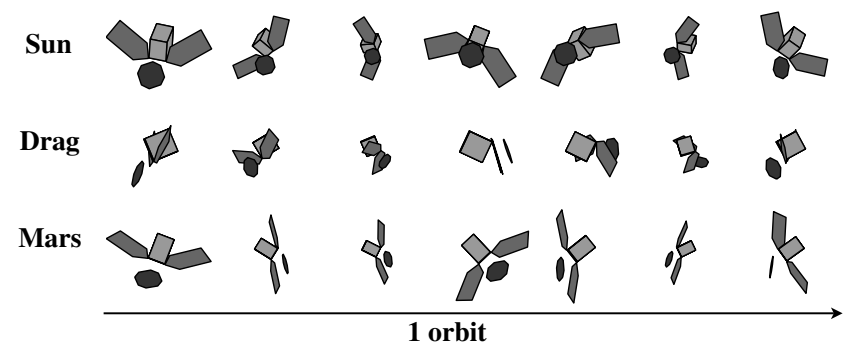

Fig. 7 Geometry of the MRO spacecraft over one orbit.

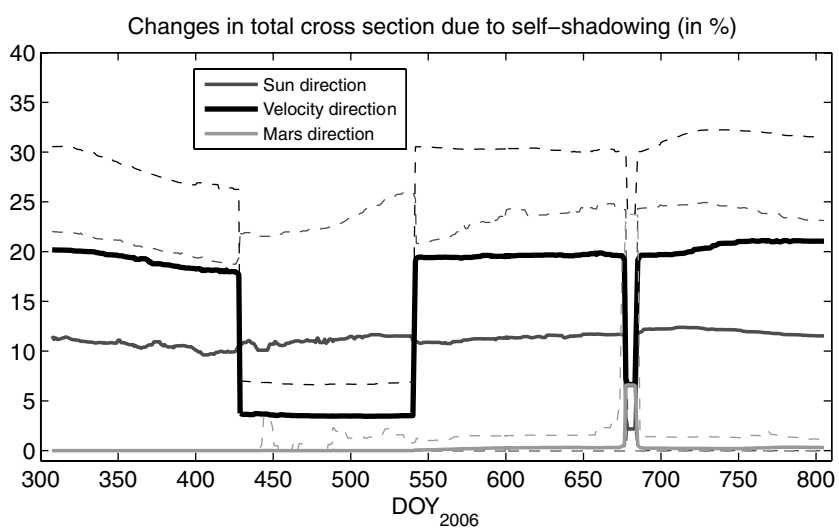

Fig. 8 Self-shadowing effect on cross section for MRO over approximately 1.5 years.

alternate attitude mode was experimented then (keeping the solar panels fixed). This generally reduced the self-shadowing, as well as the frequency of small attitude maneuvers from once every two days to once every three days.

Unlike the Mars Odyssey, cross-section changes in the velocity direction because of self-shadowing lead to orbital changes comparable to those of the radiation pressure (Fig. 9), so that both forces can be estimated more reliably. Tests were conducted where only the solar radiation benefited from the modeling improvement: the adjusted drag coefficients varied by less than $1 \%$. The average position difference between the orbits converged with and without self-shadowing included are generally small, on the order of $1 \mathrm{~m}$, but increased to several tens of meters during the major summer 2007 dust storm (Fig. 9). Without any parameter adjustment (i.e., stop the orbit determination after the first iteration), the effect is one to two orders of magnitude larger (shown in black dots with the label "1 iteration").

Similarly to Fig. 6 for the Mars Odyssey, Fig. 10 shows the adjusted drag coefficients (scaling the a priori atmospheric density) of approximately 1.5 years of MRO radio tracking data. Except for the period mentioned previously where the difference amounts to approximately $5 \%$, the inclusion of self-shadowing in the crosssection calculation changes the retrieved values, and thus the density estimates, by approximately $30 \%$. To avoid cross-section misestimates propagating to density estimates can be critical in others studies, for example, on the tidal activity in the Martian exosphere [16], as those longitudinal density variations are only approximately 4-7\% in amplitude. Note that the use of the self-shadowing algorithm for the solar radiation only (blue) does not induce changes in the obtained values, showing that the effects of the two forces are well separated by GEODYN II, certainly because they are of comparable magnitude.

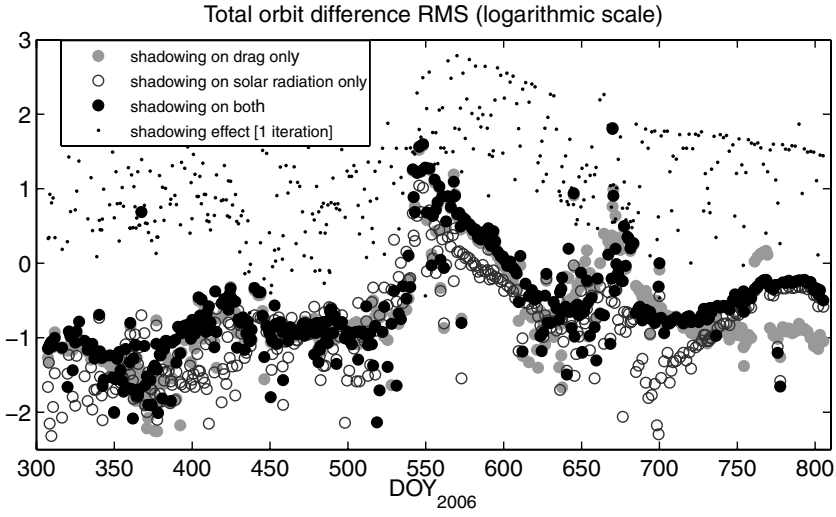

Fig. 9 Total orbit differences of the MRO arcs adjusted with different cross-section modeling strategies. 


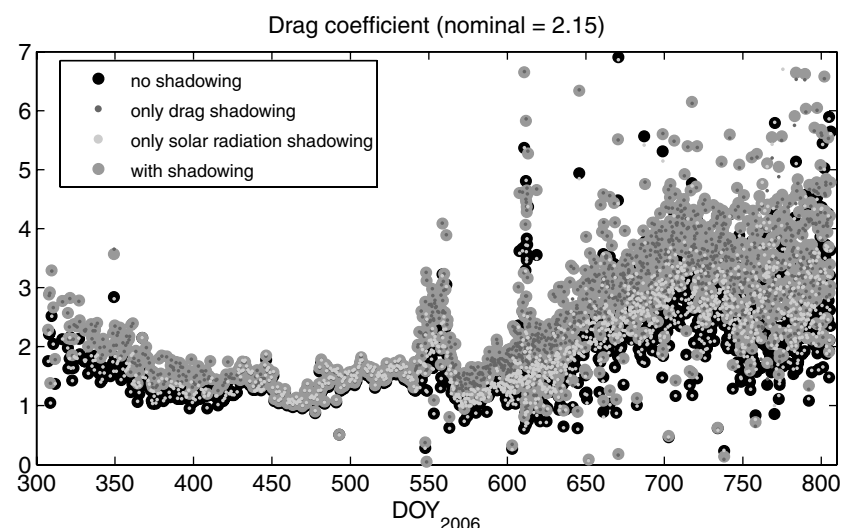

Fig. 10 Time series of the obtained drag coefficients from MRO with different modeling strategies.

\section{Current Development and Future Work}

In the case of both the Mars Odyssey and the MRO, the effect of self-shadowing on the cross section of the spacecraft viewed from nadir is negligible. This is of course due to the three-axis attitude stabilization to ensure that the scientific instruments located on the nadir side of the spacecraft bus view their target. This seems rather positive, because computationally the albedo (and thermal) radiation force is the most expensive: it is the integration of the contribution of many planetary surface elements. The precision of the albedo force modeling is directly related to the number of such elements used. In GEODYN II, the planetary surface within the horizon is divided into rings, each with a varying number of tiles (all with the same emission angle to the spacecraft) dependent on the ring radius. Typically for Mars, three rings are used, which corresponds to 70 individual surface elements. Thus, taking the self-shadowing into account for the albedo acceleration would require about 35 times more computational time than just the direct solar radiation and the atmospheric drag! This would become completely impractical.

Nevertheless, although the self-shadowing from nadir is very small, as stated previously, other directions contribute to the albedo accelerations. With the low altitude of the orbiters relative to planetary radius, the visible surface is at large off-nadir angles (approximately $63.4 \mathrm{deg}$ for the Mars Odyssey, approximately $68.7 \mathrm{deg}$ for the MRO). Thus, the self-shadowing can be expected to vary significantly, especially for a spacecraft with an asymmetric physical configuration like the Mars Odyssey.

Calculations done on the MRO with complete self-shadowing modeling show that the magnitude of the albedo acceleration changes by approximately $10 \%$ (almost constant over one orbit), its direction changes by approximately $5 \mathrm{deg}$, much more than the solar radiation (approximately $2 \mathrm{deg}$ on average) and the atmospheric drag (no change).
A solution that was explored to account for the changes in selfshadowing from the different surface elements while maintaining the overall computational load reasonable is to use spatial interpolation. For each planetary surface element, the self-shadowed cross sections of the spacecraft macromodel plates are interpolated based on a set of points whose location relative to the spacecraft are predefined. The number of surface elements can then be increased to better model the spatial variations of albedo and thermal flux, without requiring additional computationally expensive self-shadowing calculations. Figure 11 shows the good accuracy of the algorithm. On the left panel, the original map source points and interpolation nodes are shown in the projected plane (black dots and red circles, respectively). The preferred spot distribution has four rings with, respectively, $3,9,9$, and 12 nodes at radii $\frac{1}{6}, \frac{1}{3}, \frac{2}{3}$, and 1 of the maximum projected radius (corresponding to the horizon). In the center panel, the relative error (in \%) in the interpolated total cross section is plotted spatially. The interpolation is satisfactory in the visible region, and larger than average errors tend to occur in regions contributing little to the total acceleration. The white contours show the ratio of the individual node acceleration contribution to the maximum (contours are every $10 \%$; less than $50 \%$ are dotted, more than $50 \%$ thick). The errors are generally small, especially given the wide range of total spacecraft cross-section values, shown on the right panel.

In addition, in order to increase the spatial resolution of the maps used for the albedo and thermal radiations, which could be useful to model the complex asymmetrical shapes of the Martian seasonal polar caps, cylindrical gridded maps were tested instead of the spherical harmonics expansion scheme currently used in GEODYN II [10]. Rather than expanding those sets of spherical harmonics coefficients at the centers of the surface elements, simple quadratic interpolations based on the grid points were used. The computational cost of interpolation is nearly constant with increasing map resolution, whereas it grows quadratically with the spherical harmonics expansion degree.

The results of tests conducted to evaluate the computational gains are presented in Fig. 12 in terms of relative execution time cost. The new scheme of self-shadowing interpolation is not advantageous when using the spherical harmonics maps, but in all other cases it performs better. Also, the use of higher-resolution maps quickly becomes impractical if not using cross-section interpolation, with a more than 30-fold slowdown at $1 \mathrm{deg}$ resolution. It should be noted here that the interpolation algorithm used here could be further optimized for the chosen set of surface points, by precomputing the nodes to be used for interpolation depending on relative position with respect to the spacecraft.

In the case of the MRO, the albedo acceleration is the smallest of the nonconservative forces, and so the albedo modeling improvement is not warranted. In addition, due the high variability of the atmospheric density structure, the uncertainties in the atmospheric drag estimation are much greater than what can be expected to be gained in terms of orbit reconstruction quality by the improved
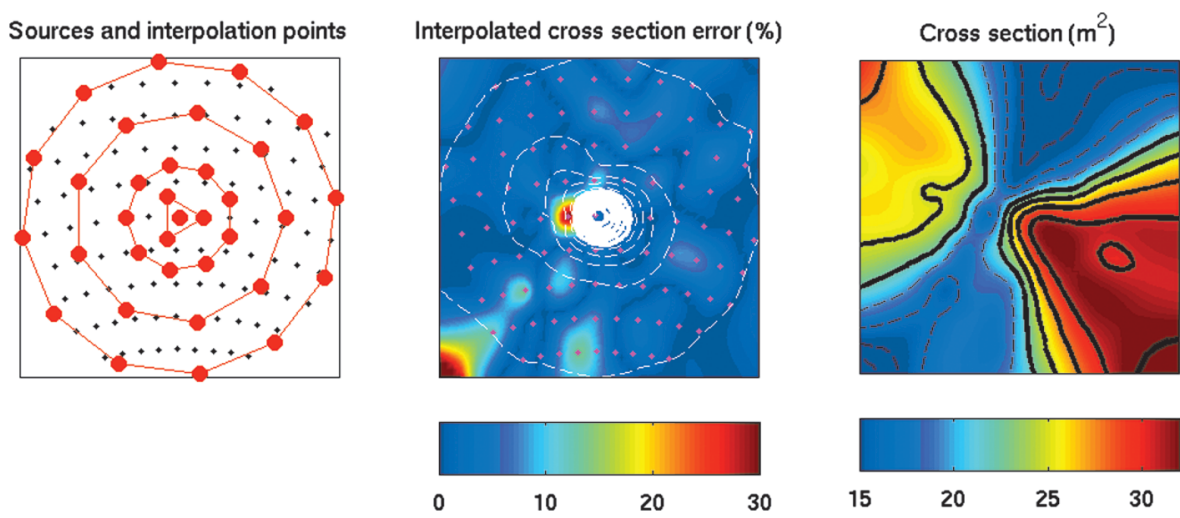

Fig. 11 Cross-section interpolation scheme and quality for the albedo radiation pressure. 
Relative computational cost of self-shadowing (\%)

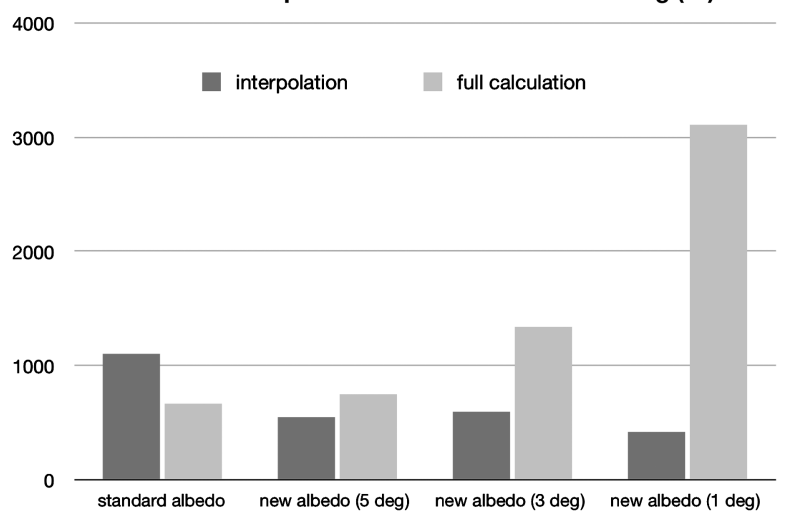

Fig. 12 Relative computational cost of using the self-shadowing algorithm.

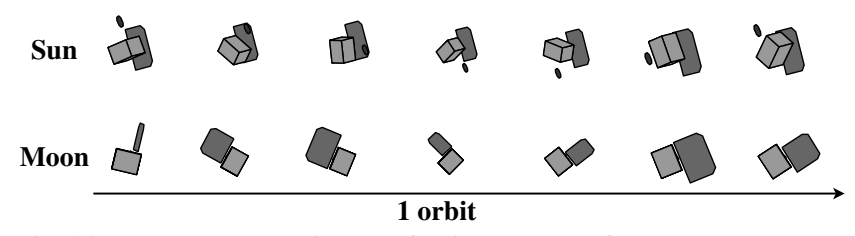

Fig. 13 Lunar Reconnaissance Orbiter spacecraft geometry over one orbit.

albedo radiation modeling. This justifies the results presented earlier (Secs. IV and V).

The focus of future work will be the POD of the Lunar Reconnaissance Orbiter $[17,18]$. The required knowledge of the spacecraft position is $50 \mathrm{~m}$, which is a challenge given the S-band tracking and the lack of any tracking data over the lunar far side. The use of altimetry crossover constraints is expected to significantly enhance the ability to reconstruct the trajectory [19], but force modeling improvements could also benefit the POD.

Compared with Mars, the albedo and thermal radiation pressure forces will be commensurate with the direct solar radiation at the mean orbiting altitude of approximately $50 \mathrm{~km}$. In addition, the estimation of the nonconservative forces will be simplified by the absence of atmospheric drag, and the radiation pressure modeling improvements will not be obscured by unknown rapid atmospheric effects. The impact of different modeling strategies on the reconstructed trajectory will be studied in test cases and expanded operationally if significant benefits are observed. Preliminary modeling of the spacecraft (Fig. 13) shows that self-shadowing will be significant for the solar radiation. Given the large size of the solar panel, it is also expected to be important for the albedo acceleration.

\section{Conclusions}

An improvement in the estimation algorithm of the spacecraft cross-sectional area is shown to be valuable both for navigation (orbit reconstruction) and scientific (parameter estimation) purposes. The impact of self-shadowing can vary significantly depending on the spacecraft physical configuration, its orbit, and the nonconservative force considered. In the case of the MRO, it is important to take selfshadowing into account for both the solar radiation pressure and the atmospheric drag, whereas for the Mars Odyssey the atmospheric drag self-shadowing has minimal effect.

The computational cost of the self-shadowing algorithm is relatively high, but this cost can be greatly mitigated by precomputation and by using those surface area values during the orbit integration. Except for testing purposes, the model improvements have not been applied to the albedo acceleration because of the added complexity and the low magnitude of that force on the Mars orbiters. Consideration of self-shadowing for the atmospheric drag and solar radiation pressure significantly improves the estimation of model parameters, in particular the atmospheric density. In the case of MRO, the changes are small but important scientifically. For the Mars Odyssey, despite periods of poor tracking geometry, the adjusted coefficients are much more reasonable than the previous, sometimes nonphysical, values.

\section{Acknowledgments}

Erwan Mazarico would like to acknowledge the following support: the NASA Mars Critical Data Products Program for his Ph.D. and his current appointment to the NASA Postdoctoral Program at Goddard Space Flight Center, administered by Oak Ridge Associated Universities through a contract with NASA.

\section{References}

[1] Yuan, D. N., Sjogren, W. L., Konopliv, A. S., and Kucinskas, A. B., "Gravity Field of Mars: A 75th Degree and Order Model," Journal of Geophysical Research, Vol. 106, No. E10, 2001, pp. $23377-$ 23401.

doi:10.1029/2000JE001302

[2] Lemoine, F. G., Smith, D. E., Rowlands, D. D., Zuber, M. T., Neumann, G. A., Chinn, D. S., and Pavlis, D. E., "An Improved Solution of the Gravity Field of Mars (GMM-2B) From Mars Global Surveyor," Journal of Geophysical Research, Vol. 106, No. E10, 2001, pp. 23359 23376.

doi:10.1029/2000JE001426

[3] Konopliv, A. S., Yoder, C. F., Standish, E. M., Yuan, D. N., and Sjogren, W. L., "A Global Solution for the Mars Static and Seasonal Gravity, Mars Orientation, Phobos and Deimos Masses, and Mars Ephemeris," Icarus, Vol. 182, No. 1, 2006, pp. 23-50. doi:10.1016/j.icarus.2005.12.025

[4] Bruinsma, S., and Lemoine, F. G., "A preliminary semi-empirical thermosphere model of Mars: DTM-Mars," Journal of Geophysical Research, Vol. 107, No. E10, 2002, pp. 5085. doi:10.1029/2001JE001508

[5] Lemoine, F. G., Bruinsma, S., Chinn, D., and Forbes, J., "Thermospheric Studies with Mars Global Surveyor," Proceedings of the AIAA/AAS Astrodynamics Specialists Conference, AIAA Paper 2006-6395, Aug. 2006.

[6] Mazarico, E., Zuber, M. T., Lemoine, F. G., and Smith, D. E., "Martian Exospheric Density Using Mars Odyssey Radio Tracking Data," Journal of Geophysical Research, Vol. 112, May 2007, p. E05014. doi:10.1029/2006JE002734

[7] Pavlis, D. E., Poulose, S. G., Deng, C., and McCarthy, J. J., "GEODYN II System Documentation," SGT-Inc., Greenbelt, MD, contractor report, 2007.

[8] King-Hele, D., Satellite Orbits in an Atmosphere: Theory and Applications, Blackie, Glasgow, 1987.

[9] Milani, A., Nobili, A. M., and Farinella, P., Non-Gravitational Perturbations and Satellite Geodesy, Adam Hilger, Bristol, England, U.K., 1987.

[10] Knocke, P. C., Ries, J. C., and Tapley, B. D., "Earth Radiation Pressure Effects on Satellites," Proceedings of the AIAA/AAS Astrodynamics Conference, AIAA, Washington, D.C., 1988, pp. 577-586.

[11] Lemoine, F. G. R., "The Dynamics of Orbiting Satellites and Gravity Model Development," Ph.D. Dissertation, Dept. of Aerospace Engineering Sciences, Univ. of Colorado, Boulder, CO, 1992.

[12] Marshall, J. A. M., and Luthcke, S. B., "Radiative Force Model Performance for TOPEX/Poseidon Precision Orbit Determination," Journal of the Astronautical Sciences, Vol. 42, No. 2, 1994, pp. 229 246.

[13] Mazarico, E., "Study of the Martian Upper Atmosphere Using Radio Tracking Data," Ph.D. Dissertation, Dept. of Earth, Atmospheric and Planetary Sciences, Massachusetts Institute of Technology, Cambridge, MA, 2008.

[14] Acton, C. H., "Ancillary Data Services of NASA's Navigation and Ancillary Information Facility," Planetary and Space Science, Vol. 44, No. 1, 1996, pp. 65-70. doi:10.1016/0032-0633(95)00107-7

[15] "SPICE Toolkit for MATLAB ${ }^{\circledR}$," http://naif.jpl.nasa.gov/naif/ toolkit_MATLAB.html (retrieved 4 Aug. 2008).

[16] Mazarico, E., Zuber, M. T., Lemoine, F. G., and Smith, D. E., "Observation of Atmospheric Tides in the Martian Exosphere Using Mars Reconnaissance Orbiter Radio Tracking Data," Geophysical Research Letters, Vol. 35, No. 9, 2008, p. L09202. doi:10.1029/2008GL033388 
[17] Chin, G., Brylow, S., Foote, M., Garvin, J., Kasper, J., Keller, J., Litvak, M., Mitrofanov, I., Paige, D., Raney, K., Robinson, M., Sanin, A., Smith, D., Spence, H., Spudis, P., Stern, S. A., and Zuber, M., "Lunar Reconnaissance Orbiter Overview: The Instrument Suite and Mission," Space Science Reviews, Vol. 129, No. 4, 2007, pp. 391-419. doi:10.1007/s11214-007-9153-y

[18] Lemoine, F. G., Rowlands, D. D., Mazarico, E., Chinn, D. S., McGarry, J., and Torrence, M. H., "Precision Orbit Determination for the Lunar Reconnaissance Orbiter," COSPAR Paper PSD1-0007-08, July 2008.
[19] Rowlands, D. D., Lemoine, F. G., Chinn, D. S., and Luthcke, S. B., "A Simulation Study of Multi-Beam Altimetry for Lunar Reconnaissance Orbiter and Other Planetary Missions," Journal of Geodesy [online journal], Nov. 2008.

doi:10.1007/s00190-008-0285-y 